\title{
Offshore metallic platforms observation using dual-polarimetric TS-X/TD-X satellite imagery: a case study in the Gulf of Mexico
}

\author{
Armando Marino, Member, IEEE, Domenico Velotto, Member, IEEE and Ferdinando Nunziata, \\ Senior Member, IEEE
}

\begin{abstract}
Satellite-based synthetic aperture radar (SAR) has been proven to be an effective tool for ship monitoring. Offshore platforms monitoring is a key topic for both safety and security of the maritime domain. However, the scientific literature oriented to the observation of offshore platforms using SAR imagery is very limited. This study is mostly focused on the analysis and understanding of the multi-polarization behavior of platforms' backscattering using dual-polarization X-band SAR imagery. This work is motivated by the fact that, under low incidence angle and moderate wind conditions, co-polarized channels may fail in detecting offshore platforms even when fine-resolution imagery are considered. This behavior has been observed on both medium and high resolution TerraSAR-X/TanDEM-X SAR imagery, despite the fact that platforms consist of large metallic structures. Hence, a simple multi-polarization model is proposed to analyze the platform backscattering. Model predictions are verified on TerraSAR-X/TanDEM-X SAR imagery, showing that for acquisitions under low incidence angle, the platforms result in a reduced co-polarized backscattered intensity even when fine resolution imagery is considered. Finally, several solutions to tackle this issue are proposed with concluding remark that the performance of offshore observation using SAR significantly improves when co-polarized channels are coherently combined.
\end{abstract}

Index Terms-SAR, target detection, polarimetry, radar, offshore platforms, maritime safety and security

\section{INTRODUCTION}

$\mathrm{T}$ ODAY oil and gas extraction is mostly onshore, however, the recent discovery of a significant number of deposits in the seabed increased the amount of offshore installations [1]. A complex infrastructure is required to drill wells, extract, process, and temporarily store crude oil and natural gas, hence for operational reasons the offshore installations were restricted to shallow waters, such as the North Sea, till the advent of deep water drilling technologies. The increased number of installations, the nature of mechanical drilling operations and extreme weather situations (e.g. hurricanes) make offshore platforms potential environmental threats. One example is the

A. Marino is with the School of Engineering and Innovation, Open University, MK7 6AA Milton Keynes, U.K. (e-mail: armando.marino@open.ac.uk).

D. Velotto is with the Maritime Safety and Security Lab, German Aerospace Center (DLR), Henrich-Focke-Str. 4, 28199 Bremen, Germany (e-mail: domenico.velotto@dlr.de). well oil blowout at the Deepwater Horizon platform drilling site in the Gulf of Mexico. Furthermore, since floating production system are dynamically positioned, they are obstacles for yachts, merchant ships and low flying airplanes creating potential threats to the safety of maritime traffic [2]. In conclusion, a continuous monitoring of offshore platforms is a matter of maritime safety and environmental security.

Traditional surveillance techniques, like coastal-based radars, flight surveys or patrol control can provide abundant information on platforms locations, but only with limited spatial and temporal coverage and at a high cost for equipment and manpower. Platforms owners have obviously all the information needed to create an updated database, but they are usually adverse in sharing this data with competitors or publically for business reasons. However, thanks to the huge development in Earth observation satellites, such information can be accessed at relatively low cost, over large areas and in a regular manner.

The monitoring of ocean metallic targets, i.e. ships and oil/gas rigs/platforms, with satellite-based synthetic aperture radar (SAR) has been proven to be effective because of radar almost all-weather and all-day acquisition capabilities [3]. In principle, any metallic target over the ocean surface is responsible for a lager backscattering, compared to the one coming from the surrounding sea surface. For this reason, offshore platforms are expected to appear in SAR intensity images as spots brighter than the background sea (see Fig 2a). Several algorithms have been developed that detect metallic targets in SAR imagery by searching for bright pixels on a darker background [4]-[10]. Among approaches based on single polarization SAR architectures, the Constant False Alarm Rate (CFAR) is the most utilized. To improve detection performance, techniques that exploit also the phase contained in Single Look Complex (SLC) SAR data have been proposed [11]-[15]. However, the information provided by backscattered intensity collected by a single-polarization SAR is not always sufficient to effectively observe metallic targets. The

F. Nunziata is with Dipartimento di Ingegneria, Università di Napoli Parthenope, Centro Direzionale isola C4, 80143 Napoli, Italia (e-mail: ferdinando.nunziata@uniparthenope.it). 


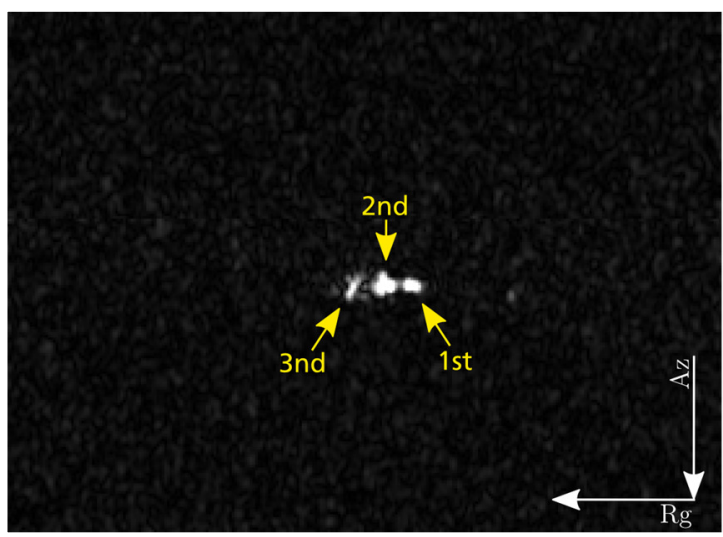

(a)

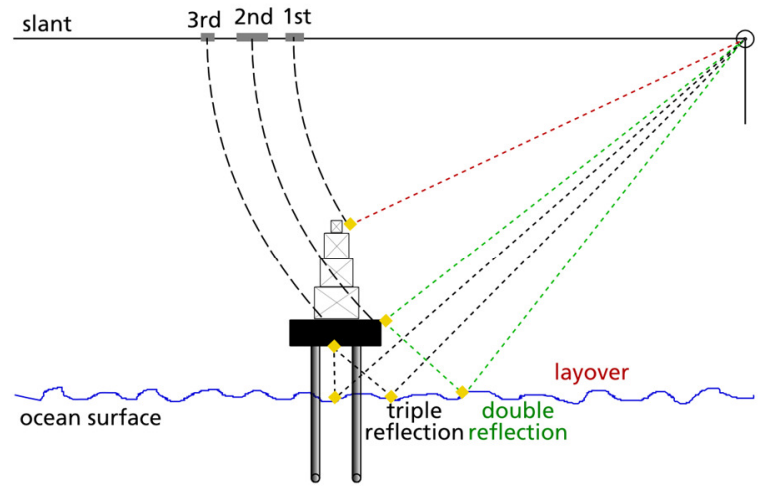

(b)

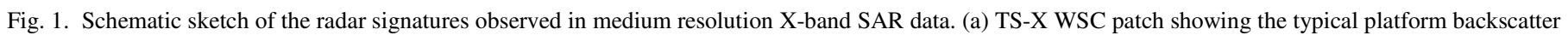
signature. (b) Schematic sketch of the different signature contributions.

availability of SAR satellites with multi polarization capabilities, hereafter PolSAR systems, triggered the development of a number of novel algorithms to detect targets at sea [16]-[25].

For the purpose of this study, it is worth mentioning that scientific literature focused on the observation of offshore platforms using SAR imagery is very limited. An example of this kind of analysis is given in [26] where the authors have built the database of platforms positions obtained by multitemporal ENVISAT ASAR acquisitions in 2008 for the North Sea area. This a priori information has then been used to quantify night-time gas flaring at offshore extractions sites by using Along Track Scanning Radiometer (ATSR). This study clearly witnesses that to ensure temporal sampling dense enough, wide swath SAR imagery is needed. In [27] fullpolarimetric SAR measurements are exploited to both observe metallic targets (exploiting combinations of co- and crosspolarized channels) and detect sea oil slicks (exploiting copolarized channels). However, full-polarimetric SAR has a limited swath coverage that may prevent its operational use for offshore platform monitoring.

\section{A. Motivations}

In this study, offshore platform monitoring is discussed using dual-polarization $\mathrm{X}$-band SAR imagery. The analysis is undertaken using a data set of TerraSAR-X/TanDEM-X (TSX/TD-X) imagery collected over a test site in Gulf of Mexico at low and high incidence angles. The motivation behind this work is the observation that, under low incidence angle (around 20 degrees) and moderate wind conditions, co-polarized channels may fail in detecting offshore platforms even when fine-resolution imagery is considered. This behavior has been observed at first in medium resolution TS-X/TD- $\mathrm{X}$ singlepolarization SAR imagery, despite the fact that platforms are large structures that extend for several tens of meters above the sea level. In order to explain such phenomenon a simple backscattering model is proposed for the platforms. Such model is then tested using dual-polarimetric TS-X/TD-X data. Finally a detection exercise is performed to show that, when copolarized channels are coherently combined, platforms can be successfully observed even at low incidence angles. This witnesses the key role played by the inter-channel phase in improving observation performance. This study is mostly focused on the observation and understanding of the polarimetric behavior of platforms and a following work will be carried out in the future that will deal with the comparison of different detectors to understand which one should be used in each acquisition conditions.

The remainder of the paper is structured as follows: in Section II the backscattering model is briefly sketched; in Section III multi-polarization platforms backscattering is analyzed using actual SAR imagery; while in Section IV detection performance is discussed using both coherent and incoherent dual-polarimetric features. Finally, conclusions are drawn in Section V.

\section{BACKSCATTERING MODEL}

To introduce the platform backscattering model, a region of interest (ROI) extracted from medium resolution X-band SAR data, that includes sea surface and the target under investigation, is shown in slant-range - azimuth coordinate in Fig. 1a. Note that the spatial resolution is $2.6 \mathrm{~m} \times 40 \mathrm{~m}$ (slantrange $x$ azimuth) and the incidence angle at the platform location is $\theta=39^{\circ} .6$. Platforms installed in shallow water consist of vertical metallic towers sustained by submersed pylons fixed to the sea floor. Fig. 1b, shows an interpretation of the different scattering contributions for a given azimuth angle. The tower's altitude can be of several tens of meters and hence it may cause several scattering mechanisms that results in multiple bright spots aligned along the range direction (indicated by the yellow arrows in Fig. 1a). The first mechanism is due to what is commonly referred as layover (dashed red line path in Fig. 1b): the direct reflection from the highest structures of the platform and it will be located before the actual position of the platform. The second mechanism is mainly caused by double reflections between the platform vertical structures and the ocean surface (dashed green line path in Fig. 1b with yellow diamonds indicating the possible point of reflections): this spot will be located vertically underneath the vertical structure. The third mechanism accounts for triple reflections (or even higher order) between the platform and the surrounding sea surface 
(dashed black line path in Fig. 1b). They could be due to the electromagnetic wave that reflects on the sea, a platform structure, again on the sea and back to the sensor (see yellow diamonds along with the dashed black path in Fig. 1b). They are located after the platform, since the path that the electromagnetic wave has to travel is longer. According to this simplistic model, these three main mechanisms make possible the detection and hence the platform monitoring using SAR data.

\section{A. Observing the backscattering of Offshore Platforms with medium resolution data}

Offshore drilling sites consist of several platforms that, when jointly connected, form super structures whose size is several tens of meters. However, in many cases offshore platforms are spread a part over the oil field; this happen for instance in Gulf of Mexico (GoM) [2]. In the case of a wide spatial distribution of platforms, the use of Scanning SAR (ScanSAR) imaging mode is a reasonable choice. With this SAR imaging mode, larger coverage is obtained at the cost of lower spatial resolution.

The TS-X/TD-X 4 beams ScanSAR (SC) mode achieves a swath width of $\sim 100 \mathrm{~km}$ at spatial resolution of $\sim 18 \mathrm{~m}$. In 2013 the TS-X/TD-X product portfolio has been extended adding a 6 beams Wide-ScanSAR (WSC) mode with $\sim 40 \mathrm{~m}$ resolution and swath width of $\sim 250 \mathrm{~km}$. Fig. 2a shows the UTM map of a projected and calibrated HH-polarized TS-X WSC amplitude image. The image was collected on August 14, 2014 at 12:08 UTC under low-moderate wind conditions $(2-5 \mathrm{~m} / \mathrm{s})$ over a cluster of offshore platforms in GoM. It is interesting to note that not all bright pixels in Fig. 2a are offshore platforms, as other marine metallic targets, e.g. ships, buoys, etc., produce a backscattered signal larger than the sea background one. To classify the bright pixels in Fig. 2a , the offshore platform records from the U.S. Bureau of Safety and Environmental Enforcement (BSEE) is merged with a cloud-free multi-spectral image collected by the Operational Land Imager (OLI) onboard the Landsat- 8 satellite. The co-located portion of the OLI image is shown in true color composite (band 4 in red, band 3 in green, band 2 in blue) in Fig. 2b. The figure is augmented with red squares, which indicate the matches between the BSEE dataset and platforms localized in the OLI sub image. A zoom-in of one of the platform $(200 \times 200$ pixels $)$ is shown in the clip on the bottom right-side of the image.

\section{B. Results of the analysis}

Comparing Fig. 2a and Fig. 2b, it is possible to conclude that most of the bright pixels in the scene of Fig. 2a are offshore platforms. Unfortunately, the physical dimensions of these targets are unknown, but the zoom-in clip of the OLI image (which has a pixel spacing of $30 \mathrm{~m}$ ) suggests these targets have dimensions larger than $30 \mathrm{~m}$. Besides, oil rigs can be several tens of meters higher than the sea level and therefore, they should be detectable in medium resolution SAR images as well (see Fig. 2 a). However, one can note that the radar backscatter of such big targets reduces significantly (apparently it vanishes) under certain incidence angles. To better clarify this point, an
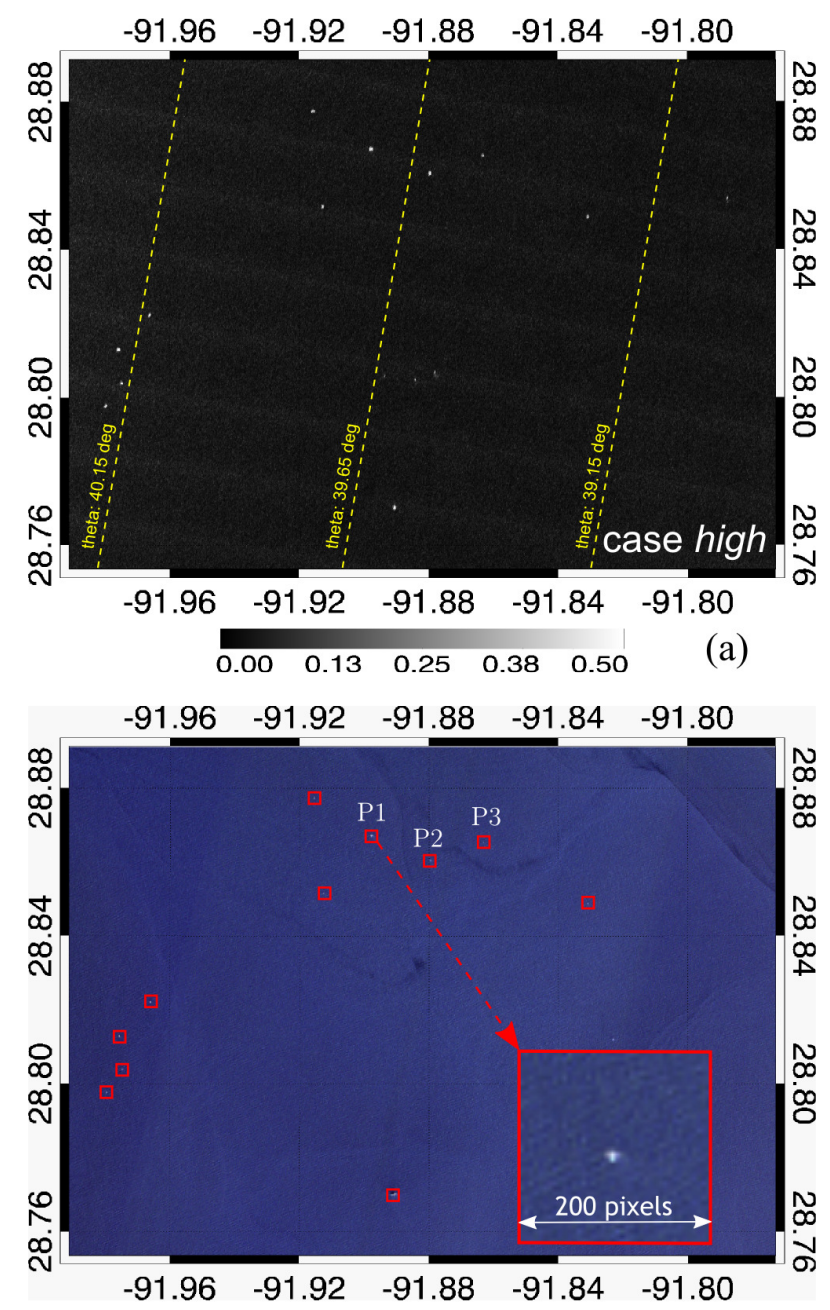

(b)

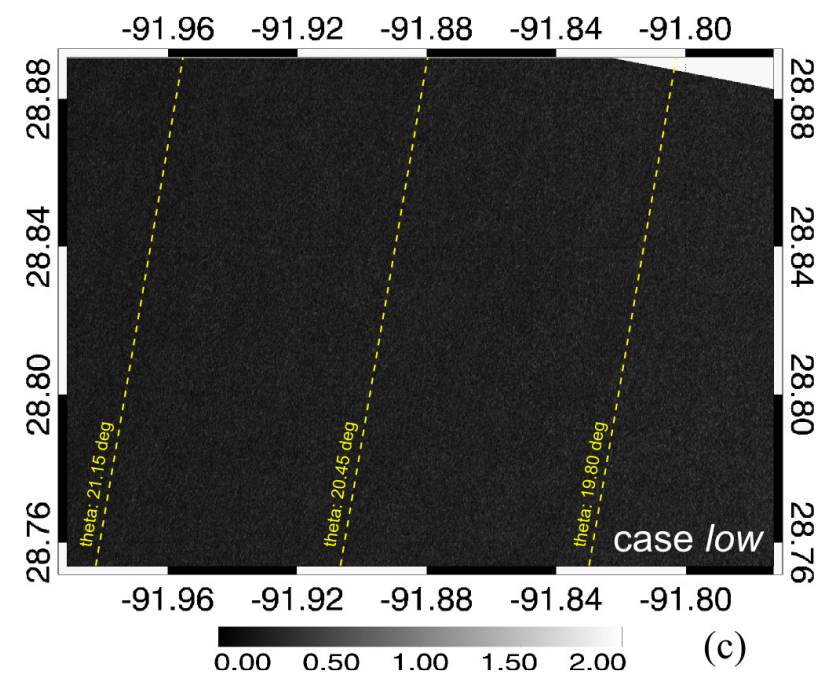

Fig. 2. Cluster of offshore platforms in GoM. (a) Map projection of the calibrated HH polarized amplitude TS-X WSC mode SAR data collected on 14 August, 2014 (case high); (b) True color composite OLI image augmented with BSEE platforms records matches; (c) Map projection of the calibrated $\mathrm{HH}$ polarized amplitude TS-X WSC mode SAR data collected on 01 May, 2014 (case low).

additional TS-X WSC scene, collected on May 01, 2014 at 12:17 UTC over the same cluster of platforms in Fig. 2a and 
TABLE I

OVERVIEW OF THE DUAL-POLARIMETRIC TS-X/TD-X SM ACQUISITIONS OVER KNOWN OFFSHORE PLATFORMS IN GOM

\begin{tabular}{cccccc}
\hline $\begin{array}{c}\text { Acquisition } \\
I D\end{array}$ & $\begin{array}{c}\text { Data } \\
\text { Time }\end{array}$ & $\begin{array}{c}\text { Resolution* } \\
R g-A z\end{array}$ & $\begin{array}{c}\text { Incidence Angle } \\
\vartheta\end{array}$ & Polarization & $\begin{array}{c}\text { Wind Speed } \\
\mathrm{m} / \mathrm{s}\end{array}$ \\
\hline GoM1 & $\begin{array}{l}2014 / 10 / 13 \\
12: 17 \mathrm{UTC}\end{array}$ & $1.2 \mathrm{~m} \times 6.6 \mathrm{~m}$ & $19.8^{\circ}-21.7^{\circ}$ & $\mathrm{HH}-\mathrm{VV}$ & $7-12$ \\
\hline GoM2 & $\begin{array}{l}2014 / 03 / 24 \\
12: 08 \mathrm{UTC}\end{array}$ & $1.2 \mathrm{~m} \times 6.6 \mathrm{~m}$ & $39.0^{\circ}-40.3^{\circ}$ & $\mathrm{HH}-\mathrm{VV}$ & $6-11$ \\
\hline GoM3 & $\begin{array}{l}2012 / 10 / 28 \\
12: 17 \mathrm{UTC}\end{array}$ & $1.2 \mathrm{~m} \times 6.6 \mathrm{~m}$ & $19.8^{\circ}-21.7^{\circ}$ & HH-HV & $8-12$ \\
\hline GoM4 & $\begin{array}{l}2014 / 03 / 02 \\
12: 08 \mathrm{UTC}\end{array}$ & $1.2 \mathrm{~m} \times 6.6 \mathrm{~m}$ & $39.0^{\circ}-40.3^{\circ}$ & HH-HV & $5-10$ \\
\hline GoM5 & $\begin{array}{l}2012 / 11 / 08 \\
12: 17 \text { UTC }\end{array}$ & $1.2 \mathrm{~m} \times 6.6 \mathrm{~m}$ & $19.8^{\circ}-21.7^{\circ}$ & VH-VV & $5-10$ \\
\hline GoM6 & $\begin{array}{l}2014 / 03 / 13 \\
12: 08 \mathrm{UTC}\end{array}$ & $1.2 \mathrm{~m} \times 6.6 \mathrm{~m}$ & $39.0^{\circ}-40.3^{\circ}$ & $\mathrm{VH}-\mathrm{VV}$ & $4-9$ \\
\hline \hline
\end{tabular}

*Nominal values. The resolution in range depends on incidence angle and increases with it.

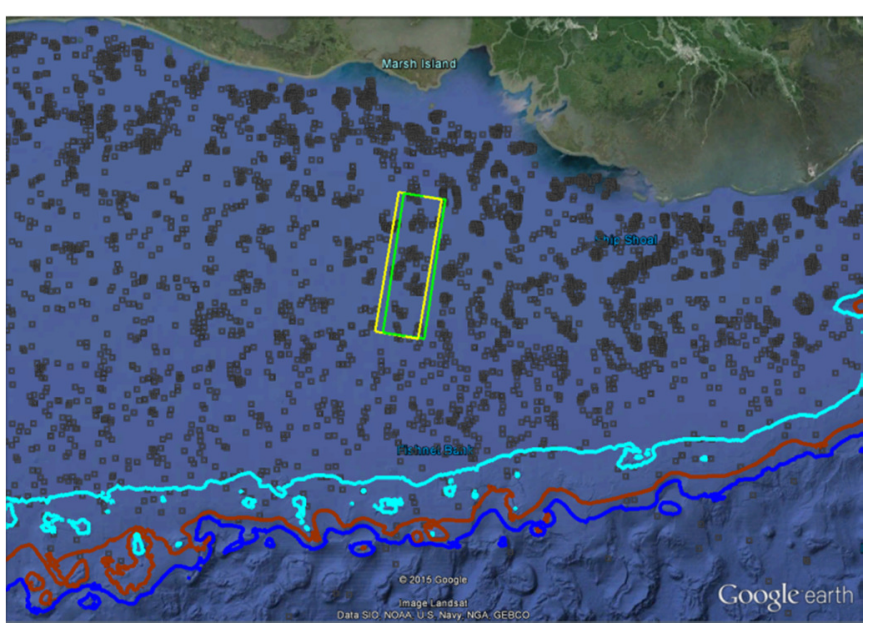

Fig. 3. Overview of the area under investigation (background @GoogleEarth). TS-X/TD-X coverages, related to low and high acquisition geometries, are shown as yellow and green rectangles, respectively. The GoM bathymetry from the NGDC Coastal Relief Model is overlaid as iso-bath for 100, 200, $300 \mathrm{~m}$ depth (cyan, red and dark blue lines respectively). Offshore platforms locations are indicated as gray rectangles.

Fig. $2 \mathrm{~b}$, is considered. The UTM projected HH polarized amplitude image is shown in Fig. 2c. Both SAR data are characterized by the same polarization, imaging mode, resolution and viewing direction. The only difference is the incidence angle, which ranges in the interval $39^{\circ} .15-40^{\circ} .15$ (case high) and $19^{\circ} .80-21^{\circ} .15$ (case low) for the scene shown in Fig. 2a and Fig. 2c, respectively. It can be noted that none of the platforms observed in Fig. 2a (and identified in Fig. 2b) results in a backscattered signal larger enough to be clearly identified in Fig. 2c. This outcome might provide an operational constraint when observing offshore platform with single polarization SAR. Therefore, deeper analysis of the radar backscatter under different polarization combinations and incidence angles is performed in the next section.

\section{OBSERVING THE BACKSCATTERING OF OFFSHORE PLATFORMS WITH HIGH RESOLUTION DATA}

In this section, a multi-polarization analysis of the signal backscattered by offshore platforms is undertaken exploiting a time series of fine resolution satellite TS-X/TD-X images collected over the same area under different incidence angles.

\section{A. Dual Polarimetric Dataset Description}

The TS-X/TD-X dataset has been collected in all possible dual-polarization combinations at two different viewing geometries using repeat pass acquisitions. All products have been acquired during satellite descending orbit (right looking) in StripMap (SM) mode which provides a nominal spatial resolution of $1.2 \mathrm{~m} \times 6.6 \mathrm{~m}$ (range $\mathrm{x}$ azimuth) and the L1b SLC data format is processed. The SAR data set is described in Table I.

This dataset consists of 3 couples (one for each dualpolarization combination) of images collected over the same cluster of platforms shown in Fig 2 at two different incidence angles that, hereinafter, are referred as low (GoM1, GoM3 and GoM5) and high (GoM2, GoM4 and GoM6).

In Fig. 3 an overview of the area under investigation is shown together with the satellite ground coverages of the low (yellow rectangle) and high (green rectangle) acquisition geometries. It can be noted that satellite coverages are almost spatially colocated and include several offshore platforms (gray dots). In addition, bathymetry information provided by the NGDC Coastal Relief Model, witnesses that platforms are located in water depth $<100 \mathrm{~m}$; hence they are Fixed type offshore platforms.

\section{B. Single-pol analysis}

To analyse the backscattering behaviour of the offshore platforms under different linear transmit/receive polarizations and with respect to the incidence angle, the four dualpolarimetric $\mathrm{HH}-\mathrm{HV}$ and $\mathrm{VH}-\mathrm{VV}$ SM TS-X/TD-X are considered, i.e. GoM3 and GoM5 for the case low and GoM4 and GoM6 for the case high. To make clearer the analysis, we will focus on three platforms randomly selected among all the platforms present. These are termed as P1, P2 and P3 in Fig. 2b. The geographical location for these platforms is provided in form of maps for an easy cross reference among the results provided in the paper.

In the following the low and high incidence angle case are treated separately:

1) The intensity images for the low incidence angle case are shown in Fig. 4. The first row of images refers to the GoM3 

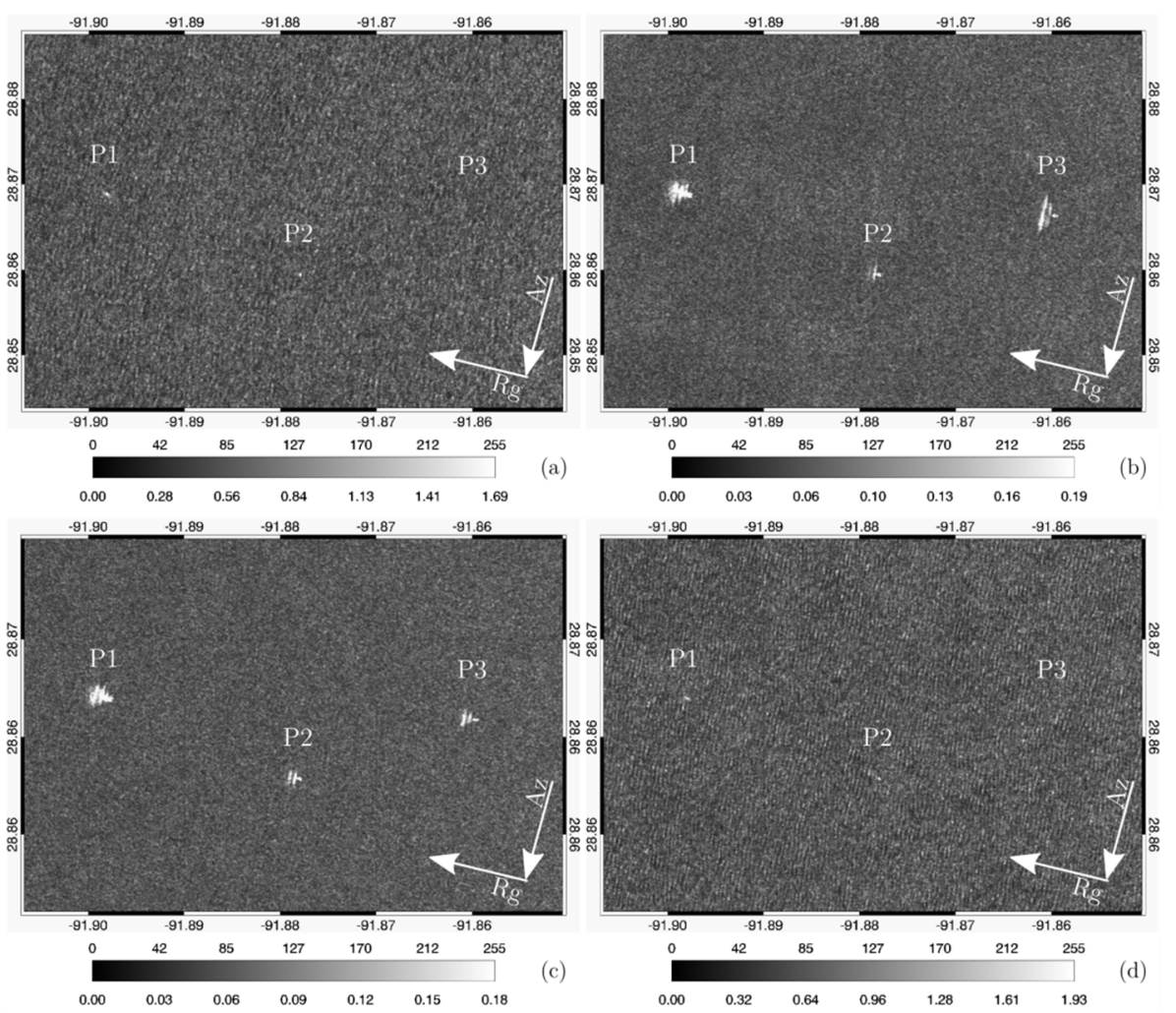

Fig. 4. Multi-temporal ground projected calibrated amplitude SAR data collected by TS-X/TD-X over a cluster of 3 offshore platforms in GoM (labeled as P1, P2 and P3). The first row shows GoM3 imagery collected at HH (a) and HV (b) polarization. The second row shows GoM5 imagery collected at VH (c) and VV (d) polarization.

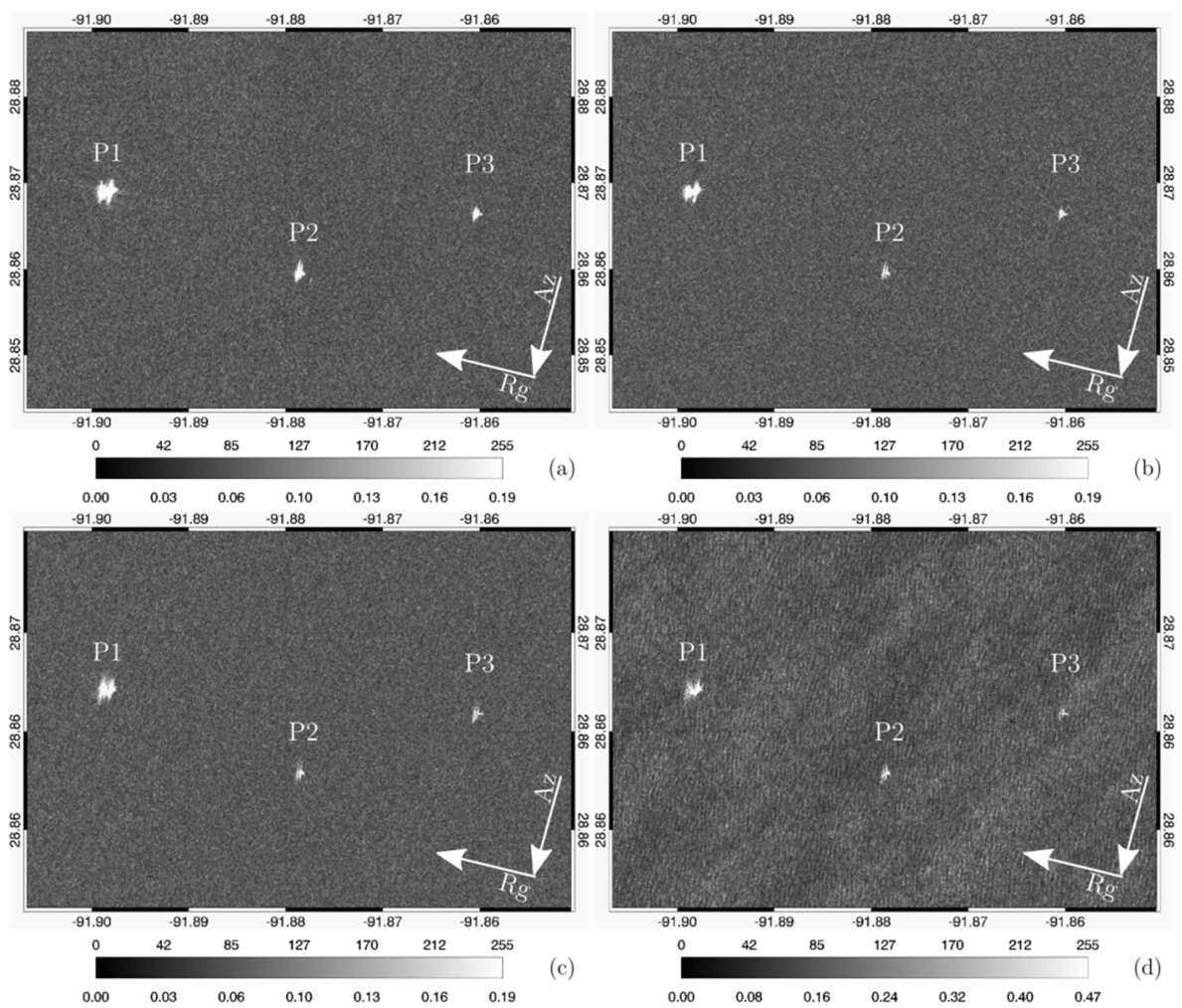

Fig. 5. Multi-temporal ground projected calibrated amplitude SAR data collected by TS-X/TD-X over a cluster of 3 offshore platforms in GoM (labeled as P1, P2 and P3). The first row shows GoM4 imagery collected at HH (a) and HV (b) polarization. The second row shows GoM6 imagery collected at VH (c) and VV (d) polarization.

acquisition, while the second row refers to the GoM5 one. All the images are ground projected and calibrated magnitudes. The figure is organised in such a way that the images on the main diagonal (Fig. 4a and Fig. 4d) refer to co-polarized HH and VV 
channels; while the off-diagonal images (Fig. 4b and Fig. 4c) refer to the cross-polarized HV and VH channels. Starting from the co-polarized backscattering, it can be observed that it is very hard to distinguish the signatures of the three platforms from the surrounding sea surface backscattering. This is especially true for P3. However, if we consider the cross-polarized channels, the platforms are well-distinguishable from the surrounding sea clutter. It is interesting to notice that if we compare the results of using high resolution images (Fig. 4) with low resolution images (Fig. 2) one can conclude that the lower spatial resolution is not playing a key role in making the co-polarized backscattering of the platform being undistinguishable from sea clutter.

2) The high incidence angle case is analyzed in Fig. 5, where the same format of Fig. 4 is adopted. In this occasion the first and second rows are referring to the acquisitions GoM4 and GoM6 respectively. It can be noted that all the platforms can be clearly distinguished from the background sea regardless the use of co- or cross-polarized channels. Interestingly, the finer spatial resolution of the SM imagery allows observing the expected signatures resulting from double and triple reflections (see Fig. 1). They appear as elongated strips oriented along the azimuth direction.

\section{Scattering mechanism analysis}

This section aims at analysing the platforms' backscattering exploiting multi-polarization SAR imagery. The images are exploited to gain some understanding on the physics of platform scattering. In particular, a physical explanation of the odd results provided by co-polarized imagery collected at low incidence angles is provided. All the information regarding the polarimetric scattering is contained in quad-polarimetric data. Unfortunately, only dual-polarization coherent SAR measurements are available. Among the dual-polarimetric channel combinations, the co-pol/co-pol ones, i.e. HH-VV, are the most informative. All the analysis conducted will be restricted to the polarimetric space that is observable using the co-polarized combination. The use of quad-polarimetric data may reveal other scattering mechanisms that we are not able to

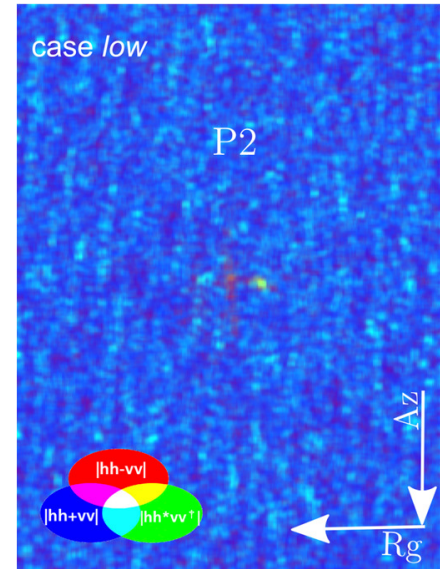

(a)

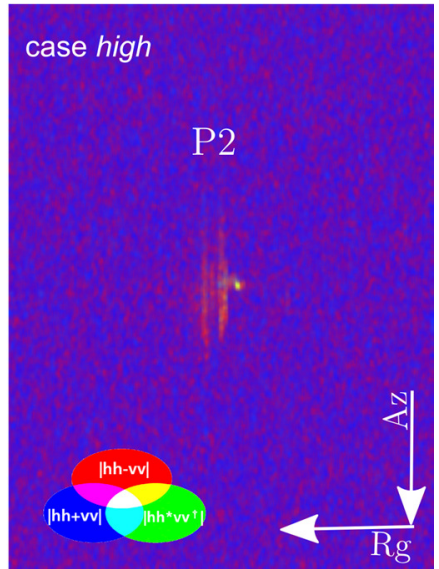

(b)

observe using only dual-polarimetric data.

The coherent HH-VV datasets GoM1 and GoM2 (see Table I) are considered and the platform P2 is used as reference. In Fig. 6a (case low) and Fig. 6b (case high) false color images are generated normalizing the all $R G B$ channels to the span of the covariance matrix. The coding used in this case is: Red = $|H H-V V|$, Green $=\left|H H *(V V)^{\dagger}\right|$ and Blue $=|H H+V V|$ (where $\dagger$ denotes complex conjugate) in order to highlight double reflection, correlation information and single reflection, respectively. In other words, for each pixel of these images, the sum of the intensity of $H H-V V$ and $H H+V V$ is unitary. The normalization process is used to get rid of the intensity information and highlight the polarimetric information content. For visualization purposes, Fig. 6c (case low) and Fig. 6d (case high) are generated without normalization but simply scaling individually the single $R G B$ channels. The coding used in this case is: Red $=|H H-V V|$, Green $=|H H+V V|$ and Blue $=$ $\left|H H *(V V)^{\dagger}\right|$. In both the cases, a $5 \times 5$ Lee filter is applied to reduce speckle noise. While the interpretation of Fig. $6 \mathrm{c}$ and Fig. 6d is straightforward, the results shown in Fig. 6a and Fig. $6 \mathrm{~b}$ deserve to be commented. The low and high incidence angle cases are, therefore, treated separately:

1) With respect to the low case (Fig. 6a), it can be noted that sea backscattering is characterized by a high single-bounce mechanism and high HH-VV coherence (bluish color) (Bragg scattering applies as expected). Platform scattering seems to show the expected three mechanisms. The rightmost part of the platform (the pixels that are closer to the sensor in range direction) is dominated by a mechanism that appears in green that represent correlation between the $\mathrm{HH}$ and VV channels. This may be a dipole scattering. The pixels in the middle of the platform are reddish which calls for a mechanism that is dihedral scattering. The dual reflection mechanism is therefore an ordinary horizontal dihedral (double-bounce). The leftmost mechanism is hard to visualize and submerged by the return from the sea.

2) With respect to the high case (Fig. 6b), sea backscattering still calls for Bragg scattering although the

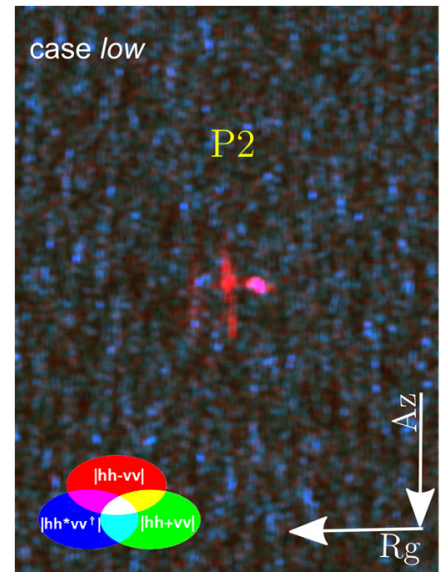

(c)

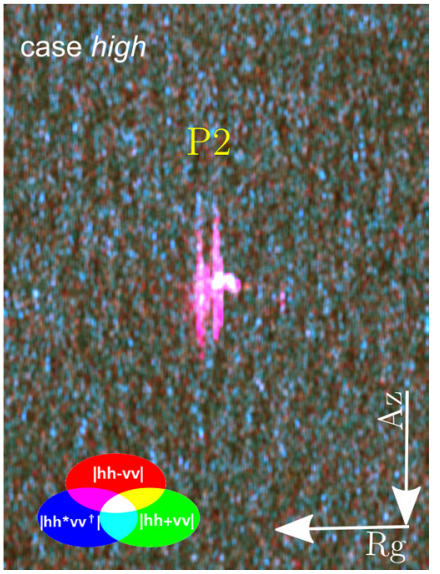

(d)

Fig. 6. False color images showing the platform P2 (see Fig 4 and Fig 5). (a) Dataset GoM1 (case low) and (b) dataset GoM2 (case high) are normalized using the span and coded as Red $=|H H-V V|$, Green $=\left|H H *(V V)^{\dagger}\right|$ and Blue $=|H H+V V|$. (c) Dataset GoM1 (case low) and (d) dataset GoM2 (case high) are scaled using the mean of each channel; Red $=|H H-V V|$, Green $=|H H+V V|$ and Blue $=\left|H H *(V V)^{\dagger}\right|$. 
pinkish color indicate a mixture with double reflection contributions. This may be due to the lower backscattering form the sea Platform backscattering clearly identifies the three mechanisms along the range direction. In fact, in this case (Fig. 6b) the rightmost part of the platform appears to be richer in red (it is more yellowish). It appears a mixture of mechanisms that could lead to a larger dihedral scattering. The pixels in the middle of the platform are reddish calling for dihedral scattering. As a matter of fact, since the reflection coefficient of the metallic platform is larger than the sea one, the platform is expected to be well distinguishable in co-polarized imagery.

To understand how much power is scattered by each mechanism we need to consider the images Fig. 6c and 6.d.

In conclusion, dihedral scattering plays an important role in platforms' backscattering. This implies that, when the incidence angle reduces, platforms are less visible in co-polarized intensity imagery since the total area of the planes representing the dihedral is reduced (because the largest plane has to be the one on the platform vertical structures). From an operational viewpoint, this means that the most critical scenario to detect offshore platforms is achieved when single-polarization copolarized, ( $\mathrm{HH}$ or $\mathrm{VV}$ ) imagery is collected at low incidence angles. Improving the spatial resolution from tens of meters (e.g. WSC mode) to meters (e.g. SM mode) does not improve platforms detectability.

Cross-polarization images are less affected by this problem because the $\mathrm{HV}$ or VH scattering is less related to the dihedral mechanism. However, cross-polarized acquisitions do not represent the standard SAR mode for geoscience applications. In fact, searching the TS-X/TD-X historical archive, about 70\% of the high resolution dual-polarimetric products are $\mathrm{HH}-\mathrm{VV}$ with the remaining $30 \%$ that includes both co-pol/cross-pol products combinations. The percentage of accessing cross-pol imagery greatly decrease (about $1 \%$ ) when medium resolution single-pol acquisitions are considered. In addition, since offshore detection is very often corroborative to sea oil pollution monitoring [28], cross-polarized channels are not the best option.

\section{APPLICATION OF DUAL-POLARIMETRIC OBSERVABLES AND DETECTORS}

Moderate wind conditions apply through the processed dataset in Table I. Therefore the sea state analyzed in this work is restricted to moderate. In the future, we will try to collect a larger dataset where we will hopefully capture different sea states. However, it is interesting to note that, even under moderate sea state conditions, may be difficult observing the SAR backscattering signature of offshore platforms. Two polarimetric detectors, namely the Geometrical Perturbation Polarimetric Notch Filter (PNF) and the Degree of Depolarization (DoD) are considered. We also tested other polarimetric observables that can be used to gain understanding of the scattering. These are the product coPro and ratio coRat of co-polarized channels. The latter are incoherent observables, i.e.; they do not exploit the inter-channel phase.

\section{A. Polarimetric observables and practical implementations}

The incoherent observables coPro and coRat are linear combination of the two measured scattering amplitudes and are therefore given by:

$$
\begin{aligned}
\text { coProd } & =|H H| *|V V| \\
\text { coRat } & =|H H| /|V V|
\end{aligned}
$$

In order to take advantage of the polarimetric information, PNF and DoD target detectors have been selected because proposed in literature as very promising and highly flexible for the detection of ships. Their performance for ship detection was shown to exceed the ones obtained via standard single-pol (either co-pol or cross-pol) detectors [18], [21], [25], [29]. It is important to note that their relevance is not limited to co-pol/copol combination. However, because the purpose of this analysis is to evaluate the effect of the lower backscattering from platforms at low incidence angle, in this work we dedicate the detection test only to co-polarization channels, where the platform are not visible and polarimetry has the potential to improve significantly the detection exercise. The application of detectors to co-pol/cross-pol products and quad-polarimetric data is left for the future, where a larger dataset will be collected to quantify the full benefits of polarimetric information.

The PNF was first proposed by Marino et. al. [18], [30] and bases the detection strategy on isolating the polarimetric signature coming from the sea and detecting anything else. For this reason, it works as a notch filter in the space of the partial targets, where the null is located on the signature of the sea.

The final detector is obtained by thresholding the polarimetric feature:

$$
\mathrm{PNF}=\frac{1}{\sqrt{1+\frac{\text { RedR }}{P_{\text {tot }}-P_{\text {sea }}}}}
$$

where $\operatorname{RedR}$ regularize the sensitivity of the distance, $P_{\text {tot }}$ and $P_{\text {sea }}$ are the total and clutter power, i.e. the difference represents the power of the target. In a practical implementation the signature of the clutter can be extracted locally using large moving windows, while the signature of the target under test can be extracted using smaller moving windows. In this study the former is extracted locally using $51 \times 51$ moving windows, the latter using $5 \times 5$ moving windows. For all experiments $\operatorname{Red} R=0.0025$ has been set. For the detection of ships in dual-polarimetric SAR data, the PNF is suggested to perform best on HH-VV combinations [18], [25], [30].

The DoD is a unitary feature, defined as inverse of the Degree of Polarization DoP, which represents the distance of the polarization state from the origin of the Poincaré sphere. Since the transmitted waves are always totally polarized, the depolarization is associated with a DoD of the incident states close to unity. As each pixel belonging to a platform can have a different polarimetric scattering mechanisms, the DoD should be higher (more depolarized) on a platform than the surrounding sea surface [21], [29], [31]. The DoD for the dualpolarimetric combination selected, is given by: 

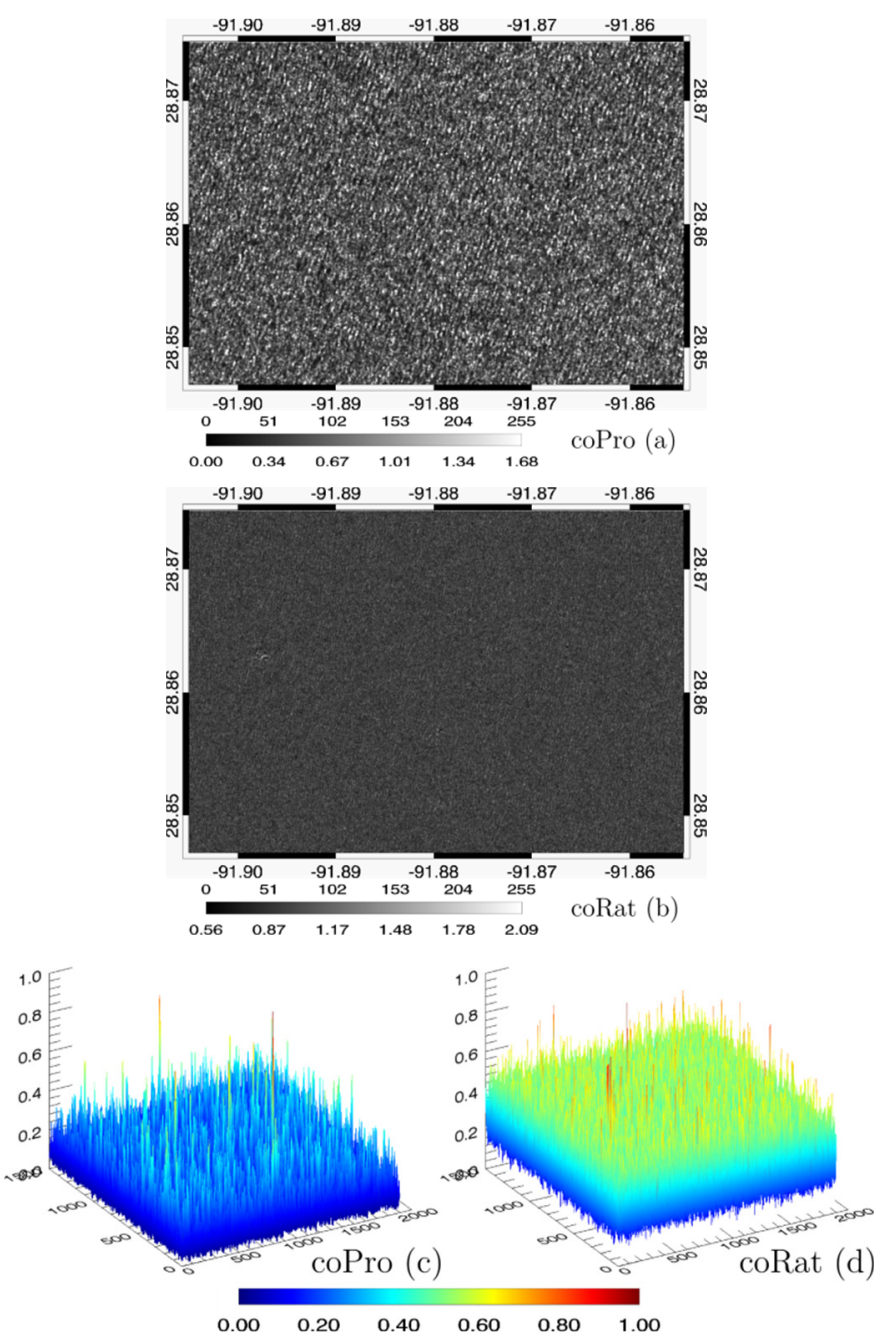

Fig. 7. Case HH-VV low incoherent analysis. (a)-(b) ground projected and byte-scaled features coPro and coRat in correspondence of the platform P1, P2 and P3; (c)-(d) Respective normalized 3D plots in satellite coordinate.

$\mathrm{DoD}=1-\frac{\sqrt{\left(|H H|^{2}-|V V|^{2}\right)^{2}+\left[2\left\langle\Re\left(H H * V V^{\dagger}\right)\right\rangle\right]^{2}+\left[2\left\langle\Im\left(H H * V V^{\dagger}\right)\right\rangle\right]^{2}}}{\left\langle|H H|^{2}+|V V|^{2}\right\rangle}$

where $\mathfrak{R}$ and $\mathfrak{S}$ denote real and imaginary part, respectively. Please note, in case of the HH-VV combination we perform an abuse of notation calling (4) "degree of depolarization", since this is applicable only when the same polarized wave is transmitted (in $\mathrm{HH}-\mathrm{VV}$ we change the polarization of the transmitted wave). The four terms in 4 are also known as Stokes parameters where the denominator is the total power and used for normalization. In this situation, the physical interpretation of the DoD is not straightforward as for the proper Stokes parameters, but it is still of value in terms of signal processing. As matter of fact in [21], DoD is suggested to perform best on $\mathrm{HH}-\mathrm{VV}$ among the linear combinations. For the processing of DoD, only a $5 \times 5$ moving windows is applied for the estimation.

\section{B. Co-pol/Co-pol case low}

Fig. 7 introduces the incoherent detection exercise for the case HH-VV low, where coPro and coRat features are displayed for the same geographical area shown in Fig. 4 and Fig. 5. For a visualization purpose, the incoherent features
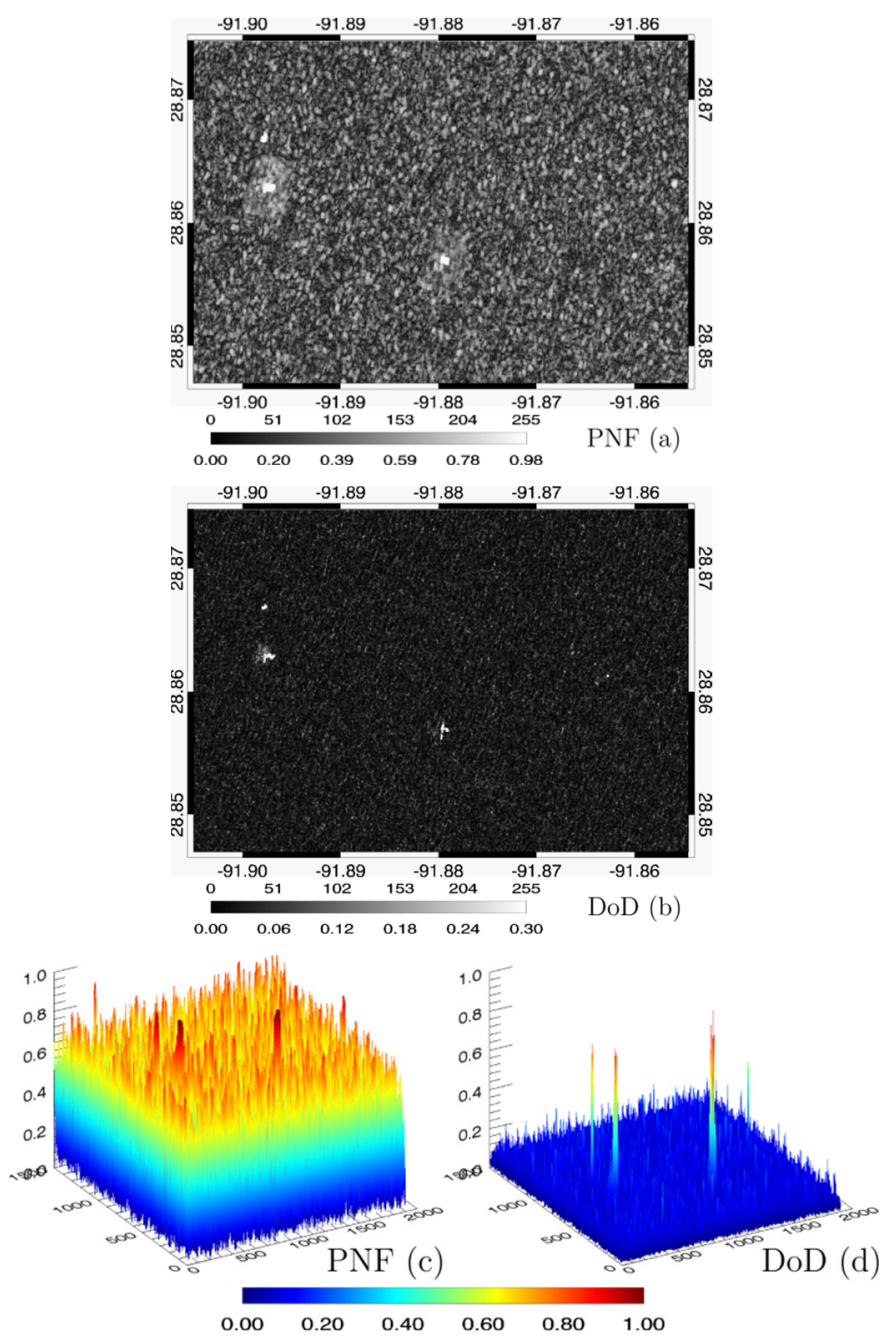

Fig. 8. Case HH-VV low coherent analysis. (a)-(b) ground projected and bytescaled features PNF and DoD in correspondence of the platform P1, P2 and P3; (c)-(d) Respective normalized 3D plots in satellite coordinate.

coPro and coRat are byte-scaled and ground projected in Fig. $7 \mathrm{a}$ and Fig. 7b. Additionally, the two features are normalized and displayed in the form of tridimensional surface in Fig. 7c and Fig. 7d. It can be noted that both incoherent features do not allow observing well-distinguishable signals associated with the platforms present. This implies that the incoherent combination (either product or ratio) do not offer a clear advantage when observing platforms at low incidence angles.

Following the same template, the coherent analysis is introduced in Fig. 8, where PNF and DoD features are considered. In this case, a completely different output is achieved that shows well-distinguishable signals associated with each of the platforms. The additional bright signature located north of the platform on the left hand side in Fig. 8a and Fig. $8 \mathrm{~b}$ is probably a passing by ship, which presence was not possible to recognize before looking at PNF and DoD outputs. Nevertheless, this is a supposition since not ground truth information about ships in the area is available. In summary these results clearly witness the added-value of coherently combining, i.e.; both amplitude and inter-channel phase, copolarized channels for platform detection application. 

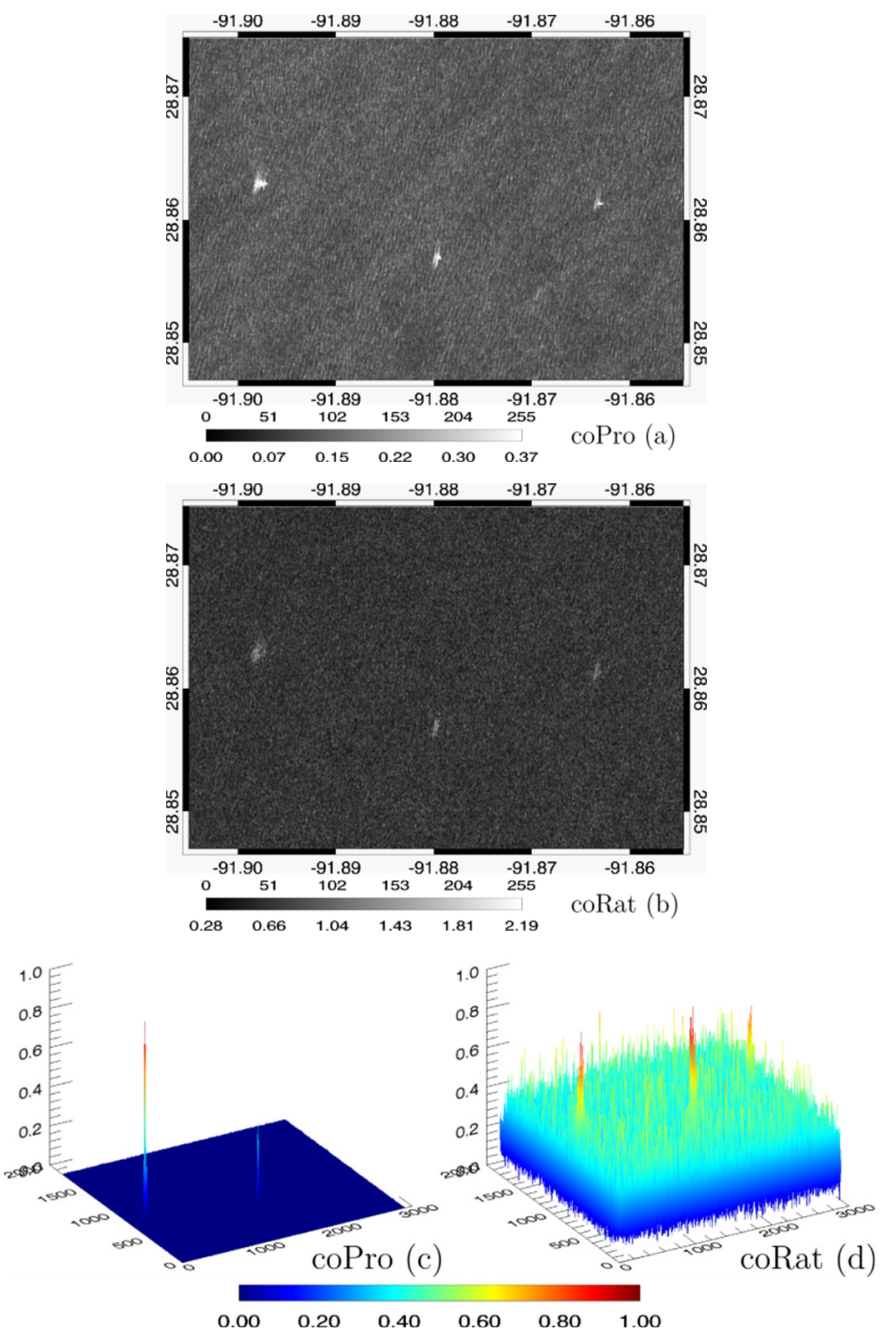

Fig. 9. Case HH-VV high incoherent analysis. (a)-(b) ground projected and byte-scaled features coPro and coRat in correspondence of the platform P1, P2 and P3; (c)-(d) Respective normalized 3D plots in satellite coordinate.

\section{Co-pol/Co-pol case high}

For a matter of completeness we show the obtained results also for the case high, although it is not as challenging as the case low. Fig. 9 introduces the incoherent analysis for the case HH-VV high, where coPro and coRat features are displayed for the same geographical area shown in Fig. 4 and Fig. 5. Similarly shows the results of the coherent analysis where PNF and DoD features are considered. In this case, both coherent and incoherent features provide well-distinguishable signals associated with the platforms considered in this investigation.

\section{CONCLUSIONS}

This study aims at analyzing satellite-based SAR observation of offshore sea platforms. A multi-polarization analysis is undertaken exploiting a data set of TerraSAR-X/TanDEM-X multi-polarization imagery. It is analyzed and discussed the multi-polarization backscattering from platforms at low (around 20 degrees) and high (around 39 degrees) incidence angles. The results obtained clearly shows that platforms, although consisting of relatively large metallic structures, may be hardly visible in single-polarization co-polarized SAR
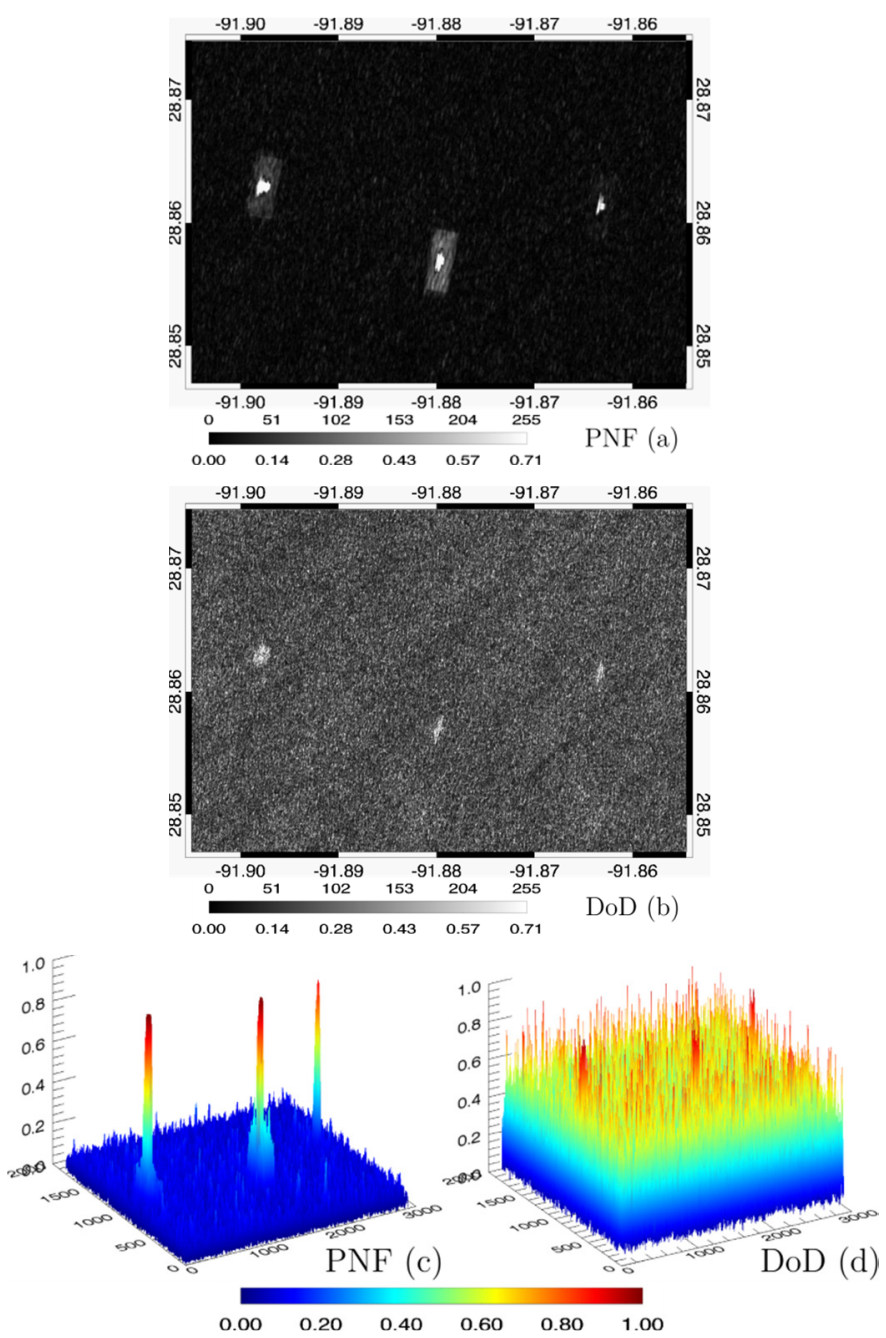

Fig. 10. Case HH-VV high coherent analysis. (a)-(b) ground projected and byte-scaled features PNF and DoD in correspondence of the platform P1, P2 and P3; (c)-(d) Respective normalized 3D plots in satellite coordinate.

imagery collected at low incidence angles under moderate sea state conditions. This phenomenon, which is explained analyzing the scattering contributions that characterize platform backscattering, is significantly mitigated when coherent dualpolarimetric co-polarized acquisitions are exploited. No improvement is obtained when incoherent dual-polarimetric copolarized combinations are exploited. Future research will address the benchmarking of different polarimetric detectors for the cases considered here including the ones that could take benefit of the cross-pol channel.

\section{ACKNOWLEDGMENT}

The authors would like to thank DLR for providing the TS$\mathrm{X} / \mathrm{TD}-\mathrm{X}$ data via the AO project (OCE1045). Offshore platform locations in the Gulf of Mexico are provided as public information by the Bureau of Safety and Environmental Enforcement. It is acknowledged that Landsat- 8 data has been downloaded from the United States Geological Survey website.

This study is partially supported by ESA-NRSCC Dragon-4 project ID 32235 entitled "Microwave satellite measurements for coastal area and extreme weather monitoring". 


\section{REFERENCES}

[1] "International Energy Agency." [Online]. Available: http://www.iea.org/. [Accessed: 18-Apr-2016].

[2] L. A. Muehlenbachs, M. A. Cohen, and T. Gerarden, "Preliminary Empirical Assessment of Offshore Production Platforms in the Gulf of Mexico," Jan. 2011.

[3] J. C. Curlander and R. N. McDonough, Synthetic Aperture Radar: Systems and Signal Processing. Wiley, 1991.

[4] D. J. Crisp, "The state-of-the-art in ship detection in Synthetic Aperture Radar imagery," May-2004. [Online]. Available: http://dspace.dsto.defence.gov.au/dspace/handle/1947/3354

[Accessed: 15-Oct-2015].

[5] S. Brusch, S. Lehner, T. Fritz, M. Soccorsi, A. Soloviev, and B. van Schie, "Ship Surveillance With TerraSAR-X," IEEE Trans. Geosci. Remote Sens., vol. 49, no. 3, pp. 1092-1103, Mar. 2011.

[6] A. Gambardella, F. Nunziata, and M. Migliaccio, "A Physical FullResolution SAR Ship Detection Filter," IEEE Geosci. Remote Sens. Lett., vol. 5, no. 4, pp. 760-763, Oct. 2008.

[7] M. Tello, C. Lopez-Martinez, and J. J. Mallorqui, "A novel algorithm for ship detection in SAR imagery based on the wavelet transform," IEEE Geosci. Remote Sens. Lett., vol. 2, no. 2, pp. 201-205, Apr. 2005.

[8] P. W. Vachon, J. W. M. Campbell, C. A. Bjerkelund, F. W. Dobson, and M. T. Rey, "Ship detection by the RADARSAT SAR: Validation of detection model predictions," Can. J. Remote Sens., vol. 23, no. 1, pp. 48-59, 1997.

[9] R. L. Paes, J. A. Lorenzzetti, and D. F. M. Gherardi, "Ship Detection Using TerraSAR-X Images in the Campos Basin (Brazil)," IEEE Geosci. Remote Sens. Lett., vol. 7, no. 3, pp. 545-548, Jul. 2010.

[10] D. Velotto, C. Bentes, B. Tings, and S. Lehner, "First Comparison of Sentinel-1 and TerraSAR-X Data in the Framework of Maritime Targets Detection: South Italy Case," IEEE J. Ocean. Eng., vol. PP, no. 99, pp. 1-14, 2016.

[11] J.-C. Souyris, C. Henry, and F. Adragna, "On the use of complex SAR image spectral analysis for target detection: assessment of polarimetry," IEEE Trans. Geosci. Remote Sens., vol. 41, no. 12, pp. 2725-2734, Dec. 2003.

[12] A. Arnaud, "Ship detection by SAR interferometry," in Geoscience and Remote Sensing Symposium, 1999. IGARSS '99 Proceedings. IEEE 1999 International, 1999, vol. 5, pp. 2616-2618 vol.5.

[13] K. Ouchi, S. Tamaki, H. Yaguchi, and M. Iehara, "Ship detection based on coherence images derived from cross correlation of multilook SAR images," IEEE Geosci. Remote Sens. Lett., vol. 1, no. 3, pp. 184-187, Jul. 2004.

[14] M. Migliaccio, F. Nunziata, A. Montuori, and R. L. Paes, "Single-look complex COSMO-SkyMed SAR data to observe metallic targets at sea," Sel. Top. Appl. Earth Obs. Remote Sens. IEEE J. Of, vol. 5, no. 3, pp. 893-901, 2012.

[15] A. Marino, M. J. Sanjuan-Ferrer, I. Hajnsek, and K. Ouchi, "Ship Detection with Spectral Analysis of Synthetic Aperture Radar: A Comparison of New and Well-Known Algorithms," Remote Sens., vol. 7, no. 5, pp. 5416-5439, Apr. 2015.

[16] C. Liu and C. H. Gierull, "A New Application for PolSAR Imagery in the Field of Moving Target Indication/Ship Detection," IEEE Trans. Geosci. Remote Sens., vol. 45, no. 11, pp. 3426-3436, Nov. 2007.

[17] G. Margarit, J. J. Mallorqui, J. Fortuny-Guasch, and C. LopezMartinez, "Phenomenological Vessel Scattering Study Based on
Simulated Inverse SAR Imagery," IEEE Trans. Geosci. Remote Sens., vol. 47, no. 4, pp. 1212-1223, Apr. 2009.

[18] A. Marino, "A Notch Filter for Ship Detection With Polarimetric SAR Data," IEEE J. Sel. Top. Appl. Earth Obs. Remote Sens., vol. 6, no. 3, pp. 1219-1232, Jun. 2013.

[19] F. Nunziata, M. Migliaccio, and C. E. Brown, "Reflection symmetry for polarimetric observation of man-made metallic targets at sea," Ocean. Eng. IEEE J. Of, vol. 37, no. 3, pp. 384-394, 2012.

[20] M. Sciotti, D. Pastina, and P. Lombardo, "Exploiting the polarimetric information for the detection of ship targets in non-homogeneous SAR images," in Geoscience and Remote Sensing Symposium, 2002. IGARSS '02. 2002 IEEE International, 2002, vol. 3, pp. 1911-1913 vol.3.

[21] R. Shirvany, M. Chabert, and J.-Y. Tourneret, "Ship and Oil-Spill Detection Using the Degree of Polarization in Linear and Hybrid/Compact Dual-Pol SAR," IEEE J. Sel. Top. Appl. Earth Obs. Remote Sens., vol. 5, no. 3, pp. 885-892, Jun. 2012.

[22] D. Velotto, M. Soccorsi, and S. Lehner, "Azimuth Ambiguities Removal for Ship Detection Using Full Polarimetric X-Band SAR Data," IEEE Trans. Geosci. Remote Sens., vol. 52, no. 1, pp. 76-88, Jan. 2014.

[23] D. Velotto, F. Nunziata, M. Migliaccio, and S. Lehner, "Dualpolarimetric terrasar-x SAR data for target at sea observation," Geosci. Remote Sens. Lett. IEEE, vol. 10, no. 5, pp. 1114-1118, 2013.

[24] C. Wang, Y. Wang, and M. Liao, "Removal of azimuth ambiguities and detection of a ship: using polarimetric airborne C-band SAR images," Int. J. Remote Sens., vol. 33, no. 10, pp. 3197-3210, May 2012.

[25] A. Marino and I. Hajnsek, "Statistical Tests for a Ship Detector Based on the Polarimetric Notch Filter," IEEE Trans. Geosci. Remote Sens., vol. 53, no. 8, pp. 4578-4595, Aug. 2015.

[26] S. Casadio, O. Arino, and A. Minchella, "Use of ATSR and SAR measurements for the monitoring and characterisation of night-time gas flaring from off-shore platforms: The North Sea test case," Remote Sens. Environ., vol. 123, pp. 175-186, Aug. 2012.

[27] M. Migliaccio, F. Nunziata, A. Montuori, X. Li, and W. G. Pichel, "A multifrequency polarimetric SAR processing chain to observe oil fields in the Gulf of Mexico," Geosci. Remote Sens. IEEE Trans. On, vol. 49, no. 12 , pp. 4729-4737, 2011

[28] X. M. Li, T. Jia, and D. Velotto, "Spatial and Temporal Variations of Oil Spills in the North Sea Observed by the Satellite Constellation of TerraSAR-X and TanDEM-X," IEEE J. Sel. Top. Appl. Earth Obs. Remote Sens., vol. PP, no. 99, pp. 1-7, 2016.

[29] F. Nunziata, A. Gambardella, and M. Migliaccio, "On the degree of polarization for SAR sea oil slick observation," ISPRS J. Photogramm. Remote Sens., vol. 78, pp. 41-49, Apr. 2013.

[30] A. Marino, M. Sugimoto, K. Ouchi, and I. Hajnsek, "Validating a Notch Filter for Detection of Targets at Sea With ALOS-PALSAR Data: Tokyo Bay," IEEE J. Sel. Top. Appl. Earth Obs. Remote Sens., vol. 7, no. 12, pp. 4907-4918, Dec. 2014.

[31] J. J. Van Zyl, "Unsupervised classification of scattering behavior using radar polarimetry data," Geosci. Remote Sens. IEEE Trans. On, vol. 27, no. 1, pp. 36-45, 1989. 